\title{
Radiological manifestations, histological features and surgical outcomes of pulmonary meningothelial proliferation: a case series and rethinking
}

\author{
Dong Lin ${ }^{1}$, Yangli Yu ${ }^{2}$, Hao Wang ${ }^{1}$, Yong Fang ${ }^{1}$, Jun Yin ${ }^{1}$, Yaxing Shen ${ }^{1}$, Lijie Tan ${ }^{1}$ \\ ${ }^{1}$ Department of Thoracic Surgery, ${ }^{2}$ Department of Radiology, Zhongshan Hospital Fudan University, Shanghai, China \\ Contributions: (I) Conception and design: L Tan; (II) Administrative support: L Tan, Y Shen, H Wang; (III) Provision of study materials or patients: \\ All authors; (IV) Collection and assembly of data: D Lin, Y Yu; (V) Data analysis and interpretation: All authors; (VI) Manuscript writing: All authors; \\ (VII) Final approval of manuscript: All authors. \\ Correspondence to: Lijie Tan, MD, FACS. Department of Thoracic Surgery, Zhongshan Hospital of Fudan University, No. 180 Fenglin Rd., Shanghai, \\ China. Email: tan.lijie@zs-hospital.sh.cn.
}

\begin{abstract}
Background: Lung cancer screening with computed tomography (CT) has been adopted worldwide. Consequently, there is an increase of some previously rarely reported lesions, like pleuropulmonary meningothelial proliferation (PMP), which included metastatic pulmonary meningiomas (MPMs), primary pulmonary meningiomas (PPMs) and minute pulmonary meningothelial-like nodules (MPMNs). To date, the understanding of the clinical management of PMP is limited.

Methods: We retrospectively searched PMP (MPMs, PPMs, and MPMNs) on the medical database (May 2005 to April 2019) of our department, and extracted the demographic and perioperative information. The last follow-up was in May 2019.

Results: A total of 78 patients, including 16 (20.5\%) males and 62 (79.5\%) females, were enrolled, with the median age as 61 (range, 27-78) years. There was 1 case with multiple MPMs, 4 cases with PPMs and 73 cases with MPMNs. On CT, MPMs manifested as round nodules with solid or part-solid components. PPM mainly presented as an isolated, round, solid, and well-circumscribed nodule, with or without lobulation. While MPMNs often showed as multiple, round, randomly-distributed, solid or partly-solid nodules, most of which were in tiny size. Histologically, MPM, PPM, and MPMN had similar morphological and immunohistochemical (IHC) features. As to the surgical outcomes, the case with multiple MPMs had undergone a progression after wedge resection and was in an active monitoring. And the four cases with PPMs and the five cases with MPMNs were all in regular surveillance.

Conclusions: PPM has variable radiological manifestations. But it can be histologically identified by the characteristic features. Although surgery is able to achieve satisfying prognosis, active observation is an alternative in selected cases due to the surgical invasiveness and the indolent nature of the disease.
\end{abstract}

Keywords: Pulmonary meningothelial proliferation; radiology; histology; surgery

Submitted Dec 21, 2019. Accepted for publication Jun 10, 2020.

doi: $10.21037 /$ tlcr-19-699

View this article at: http://dx.doi.org/10.21037/tlcr-19-699

\section{Introduction}

Lung cancer is the leading cause of mortality in the world with more than 1.7 million deaths annually (1). Screening with low-dose or thin-slice computed tomography (CT) has been widely adopted for early detection and intervention $(2,3)$. As a consequence, in recent years, there is a rapid increase in the detection of accidental pleuropulmonary nodules (4), including some rare lesions, like pleuropulmonary meningothelial proliferation (PMP).

In this study, the umbrella term as PMP contains 
(I) metastatic pulmonary meningiomas (MPMs), (II) primary pulmonary meningiomas (PPMs) and (III) minute pulmonary meningothelial-like nodules (MPMNs). Previous studies suggested that these lesions could share some similar genetic, histological and biological features (5-8). To date, however, the understanding of the clinical management of PMP is limited.

Metastatic meningioma was first reported in 1880s (9). And lung is one of the most frequently metastatic sites (10). So far, there is little evidence on the optimal intervention for MPM, especially for multiple lesions (11). PPM, as a rare entity, is usually considered as a benign tumor with indolent course (12). However, it could also have metastasis $(13,14)$. The change in the term, from pulmonary paraganglioma (chemodectoma) (15) to MPMNs (16), is resulted from the recognition of the microstructure of the tiny lesions. The incidence of MPMNs is much higher than those of MPMs and PPMs, reaching $0.3 \%$ to $13.8 \%$ among cases of autopsy and resection (6,17-19). MPMNs often manifest as multiple, round, solid or partly-solid nodules less than $5 \mathrm{~mm}$ in diameter (17-19). It has raised more and more interest with the increase of early lung cancer presenting as small nodules.

In this study, we retrospectively reviewed a series of PMP, and conducted a comprehensive presentation of the radiological manifestations, histological features and surgical outcomes. We aimed to improve the understanding of the clinical management of PMP.

\section{Methods}

\section{Patients}

We retrospectively searched PMP (MPMs, PPMs and MPMNs) on medical database (May 2005 to April 2019) of Department of Thoracic Surgery, Zhongshan Hospital, Fudan University. All of these patients had undergone surgical resection and pathological examination.

\section{Data collection}

The demographic information was extracted from the database. And the perioperative data, mainly including medical imaging, surgical information, immunohistochemical (IHC) staining, were extracted from the electronic medical system. The follow-up data were attained by multiple approaches, including outpatient clinic and phone calls. The last follow-up was in May 2019. All the data were secured. Each patient had signed the informed consent form and approved the usage of the information at the initial administration. The institutional review board of Zhongshan Hospital of Fudan University approved this study (HGBB-20191201). The study was conducted in accordance with the Declaration of Helsinki.

\section{Statistical methods}

As a case series study, no statistical comparison was conducted. The continuous data was presented as mean with standard deviation (SD) or median with ranges, and the categorical data was presented as numbers with proportion (\%). In this study, the surveillance period before surgery was defined as the interval between the date of the first detection and the date of surgery; the surveillance period after surgery was defined as the interval between the date of the surgery and the data of the last follow-up.

\section{Results}

\section{Baseline characteristics}

A total of 78 patients, including 16 (20.5\%) males and 62 $(79.5 \%)$ females, were enrolled. There was 1 case with MPMs, 4 cases with PPMs and 73 cases with MPMNs. The median age was 61 (range, 27-78) years. Most of the patients were non-smokers $(73,93.6 \%)$ and had no respiratory diseases $(77,98.7 \%)$. The clinical information was presented in Table 1.

\section{Radiological, surgical and histological information}

\section{MPMs}

The patient with MPMs was asymptomatic. The disease was initially found on a chest X-ray before the operation for intracranial meningioma in July 2011. The subsequent CT revealed that there were multiple pulmonary nodules and one mediastinal mass. The maximal pulmonary nodule was located in the right lower lobe, measured as $10 \mathrm{~mm}$ with a round, solid and well-circumscribed appearance (Figure 1A). There were several similar but smaller nodules in the bilateral lobes, ranging from 1 to $6 \mathrm{~mm}$ (Figure $1 \mathrm{~B}$ ). On the ${ }^{18}$ F-FDG PET-CT scan in the same period, none of the nodules showed abnormal metabolic activity while the mediastinal mass measured as $13 \mathrm{~mm}$ had a slight uptake (Figure 1C). Then in July 2016, the patient underwent a wedge resection of one peripheral pulmonary lesion due to 
Table 1 Clinical characteristics of patients with MPM, PPM and MPMN

\begin{tabular}{|c|c|c|c|}
\hline Characteristics & MPMs $(n=1)$ & PPMs $(n=4)$ & MPMNs $(n=73)$ \\
\hline $\begin{array}{l}\text { Median age } \\
\text { [range], yrs }\end{array}$ & 62 & $60[53-72]$ & $61[27-78]$ \\
\hline \multicolumn{4}{|l|}{ Gender } \\
\hline Female & 1 & $3(75.0)$ & $58(79.5)$ \\
\hline Male & 0 & $1(25.0)$ & $15(20.5)$ \\
\hline \multicolumn{4}{|l|}{ Smoking history } \\
\hline Yes & 0 & $2(50.0)$ & $3(4.1)$ \\
\hline \multicolumn{4}{|l|}{ Respiratory diseases } \\
\hline Tuberculosis & 0 & $1(25.0)$ & 0 \\
\hline Pneumonia & 0 & 0 & 0 \\
\hline \multicolumn{4}{|l|}{ Diabetes mellitus } \\
\hline Yes & 0 & $1(25.0)$ & $6(8.2)$ \\
\hline \multicolumn{4}{|c|}{ Cardiovascular diseases } \\
\hline Hypertension & 1 & 0 & $30(41.1)$ \\
\hline $\begin{array}{l}\text { Coronary artery } \\
\text { diseases }\end{array}$ & 0 & 0 & $2(2.7)$ \\
\hline Cardiac arrhythmia & 0 & 0 & $2(2.7)$ \\
\hline \multicolumn{4}{|c|}{ Intracranial meningioma } \\
\hline Yes & 1 & 0 & $1(1.4)$ \\
\hline \multicolumn{4}{|l|}{$\begin{array}{l}\text { History of } \\
\text { malignancy }\end{array}$} \\
\hline Yes & 0 & $1(25.0)$ & $10(13.7)$ \\
\hline
\end{tabular}

MPM, metastatic pulmonary meningioma; PPM, primary pulmonary meningioma; MPMN, minute pulmonary meningothelial-like nodule.

the gradual increase in diameters of the nodules. And the lesion was histologically confirmed as MPM. During the subsequent surveillance, the CT scan in December 2018 revealed that the maximal pulmonary nodule in the right lower lobe had increased to $19 \mathrm{~mm}$ (Figure 1D) and the mediastinal mass had increased to $43 \mathrm{~mm}$ (Figure 1E).

Histologically, MPM showed as spindle cells organized in bundles and whorls (Figure $2 A$, hematoxylin-eosin, $\times 200)$ and positive staining for Vimentin (Figure $2 B, \times 100$ ), EMA (Figure $2 C, \times 200)$ and PR (Figure $2 D, \times 200)$ in IHC examination.

To date, the patient received an active monitoring but no intervention on the pulmonary lesions and the mediastinal mass.

\section{PPMs}

The four patients with PPMs also had no specific symptoms. On chest CT scan, the PPM appeared as an isolated, round, solid, and well-circumscribed nodule, with or without lobulation (Figure $3 A, B$ ). For the four patients, the brain magnetic resonance imaging scan found no absence of intracranial meningioma. None of the patients received ${ }^{18}$ F-FDG PET-CT before operation.

Notably, one of the patients underwent a percutaneous puncture and histological examination in local hospital. After resection, macroscopic and IHC examination of the specimen revealed spindle cells arranged in swirls (Figure $4 A$, hematoxylin-eosin, $\times 200)$ and positive staining for Vimentin (Figure $4 B, \times 100$ ), EMA (Figure $4 C, \times 200$ ) and PR (Figure $4 D$, $\times 200)$. As to the IHC examination, it was positive for EMA (4/4,100\%), Vimentin (4/4, 100\%), Ki-67 (3/3, 100\%), CD68 (1/1, 100\%), PR (3/4, 75\%) and CD34 (2/3, 66.7\%) (Table 2).

To date, all of four patients were alive with the longest follow-up reaching 14 years.

\section{MPMNs}

In this retrospective study, 73 cases were identified with MPMNs. Of these patients, 68 (93.2\%) had MPMNs with synchronous lung cancer-related lesions, including atypical adenomatous hyperplasia, adenocarcinoma in situ, minimally invasive adenocarcinoma and invasive adenocarcinoma. These MPMNs manifested on chest CT scan as multiple, round, randomly-distributed, solid or partly-solid nodules, most of which were less than $4 \mathrm{~mm}$.

For the other 5 (6.8\%) patients without synchronous lung cancer-related lesions, however, the MPMNs were initially presumed as pulmonary malignancies, and thus underwent surgical resection. These MPMNs were shown on chest CT as ground-glass opacities (GGOs) with or without mixed components (Figure $5 A, B, C$ ), or solid nodule (Figure $5 D$ ), measured from 5 to $14 \mathrm{~mm}$, mimicking early lung cancer.

Among these five cases, before surgical intervention, three patients had a long-term surveillance (ranging from 2 to 6 years), which suggested little progression no matter in diameter or density; the other two were radiologically diagnosed with malignant lesions due to the large sizes and the solid components.

After surgery, macroscopic examination revealed similar features as metastatic or PPM (Figure 6A). A total of 35 cases with 38 lesions received IHC examination, which 

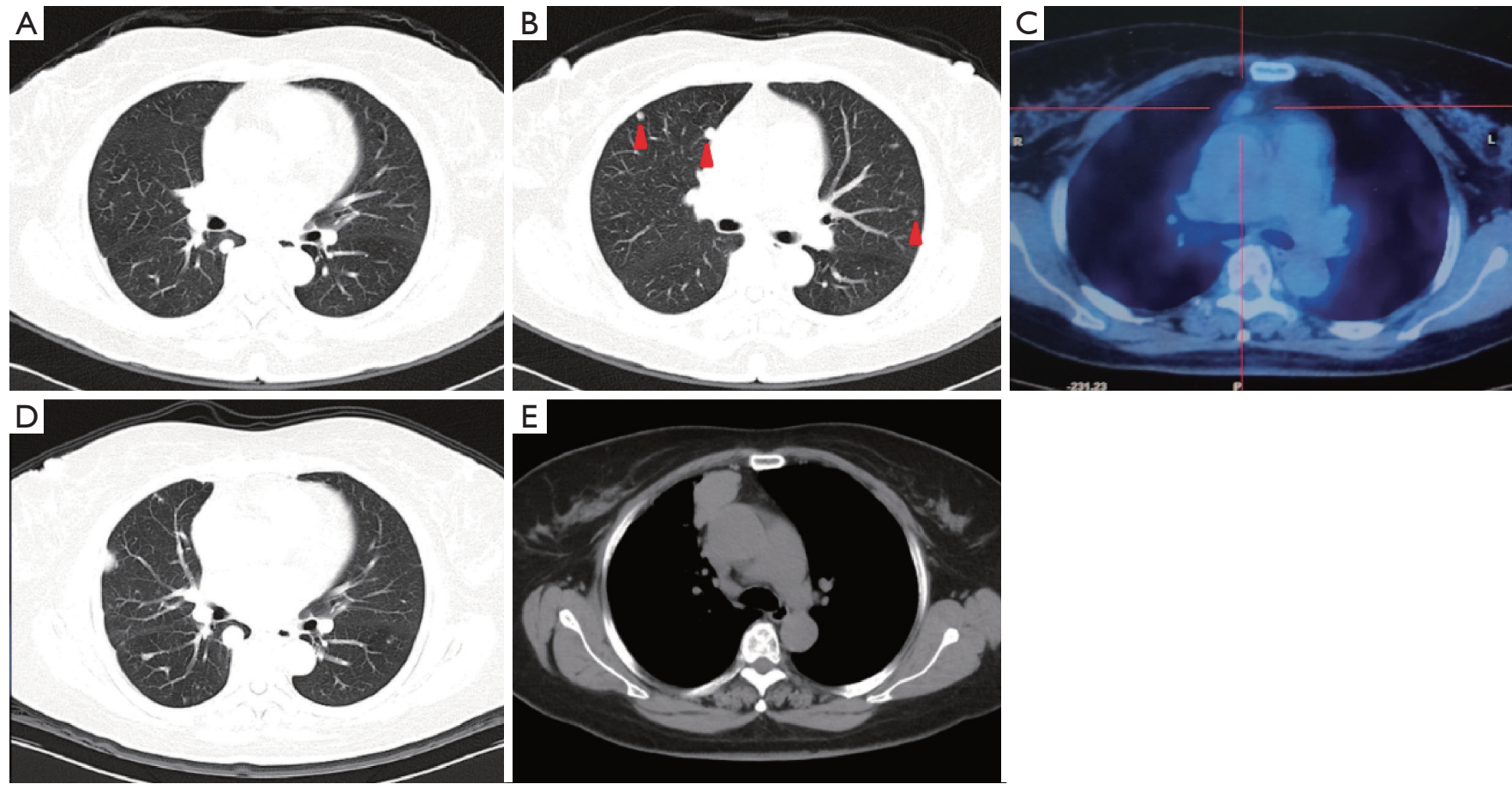

Figure 1 MPMs on positron emission tomography with or without CT. (A) the pulmonary nodule in the right lower lobe at the first detection; (B) the smaller nodules in bilateral lobes; (C) the metabolic uptake of the mediastinal mass; (D) the increasement of the nodule in the right lower lobe after 7-year' surveillance; (E) the increasement of the mediastinal mass after 7-year' surveillance. Arrows: the smaller nodules in bilateral lobes. MPMs, metastatic pulmonary meningiomas; CT, computed tomography.

comprehensively suggested that it was positive for Vimentin (Figure 6B, 12/13, 92.3\%,), EMA (Figure 6C, 37/37,100\%), PR (Figure 6D, 30/35, 85.7\%), Ki-67 (15/15, 100\%), CD68 $(5 / 5,100 \%)$ and CD34 (5/6, 83.3\%) (Table 2).

There were no recurrences of MPMNs after resection. The five patients only with MPMNs were all in a regular surveillance.

\section{Discussion}

In this study, the umbrella term as PMP included MPMs, PPMs and MPMNs. According to the published studies, these lesions usually caused no specific symptoms and were identified incidentally. Currently, the understanding of the clinical management of PMP is limited. We retrospectively reviewed the case series in our center and presented radiological manifestations, histological features, and surgical outcomes. All of these made us rethink the clinical management of the disease.

As previously reported, MPMs usually presented as isolated or multiple, round and well-circumscribed nodule, located within the lobes or nearby the pleura $(20,21)$. The clinical diagnosis was usually based on the history of intracranial meningioma. But it could be difficult as the absence of intracranial meningioma. In one particular case, an incidental pulmonary meningioma histologically confirmed after surgery revealed an unexpected intracranial meningioma (22). For resectable MPMs, surgical intervention could achieve satisfying long-term survival (21), which suggested that it tended to be benign in spite of "metastatic" nature. However, for the cases with unresectable extensive metastasis, the treatment could be intractable. Some retrospective and small prospective study suggested that chemotherapy and antiangiogenic therapy could have some roles $(11,23,24)$. Other novel systemic therapies are still on the way. In our study, the patient has received an active observation for three years. And the lesions, both the pulmonary and the mediastinal ones, are gradually progressing. From our point of view, a radical resection of the lesions causing symptoms, with or without subsequent medical therapy, may be appropriate.

PPMs had a diverse appearance on chest CT. It usually 

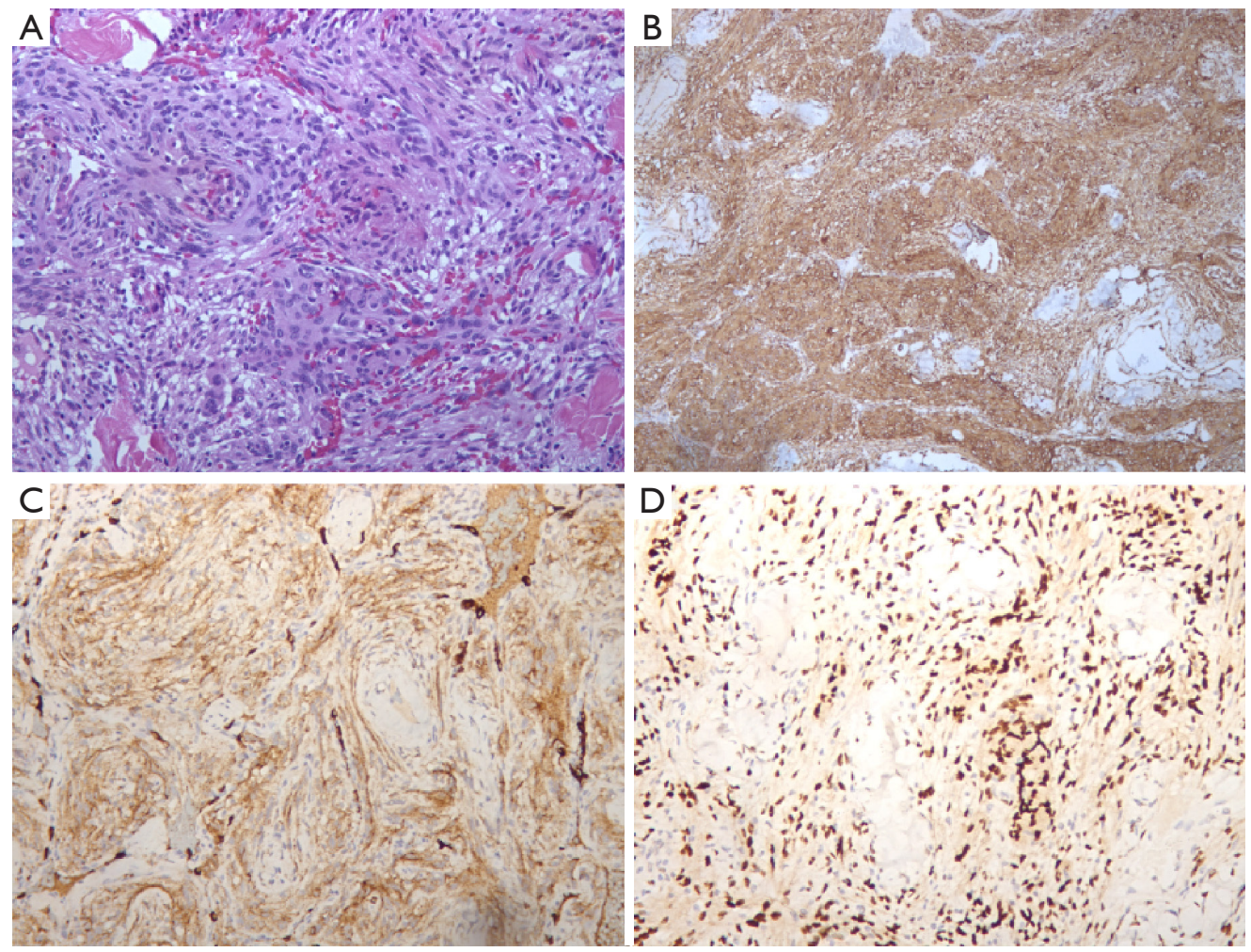

Figure 2 Histological features of MPMs. (A) Macroscopically, MPMs showed as spindle cells arranged in swirls on hematoxylin-eosin staining ( $\times 200)$; (B,C,D) immunohistochemically, metastatic pulmonary meningioma showed positive for Vimentin $(\times 100)$, EMA $(\times 200)$, PR (×200). MPMs, metastatic pulmonary meningiomas.

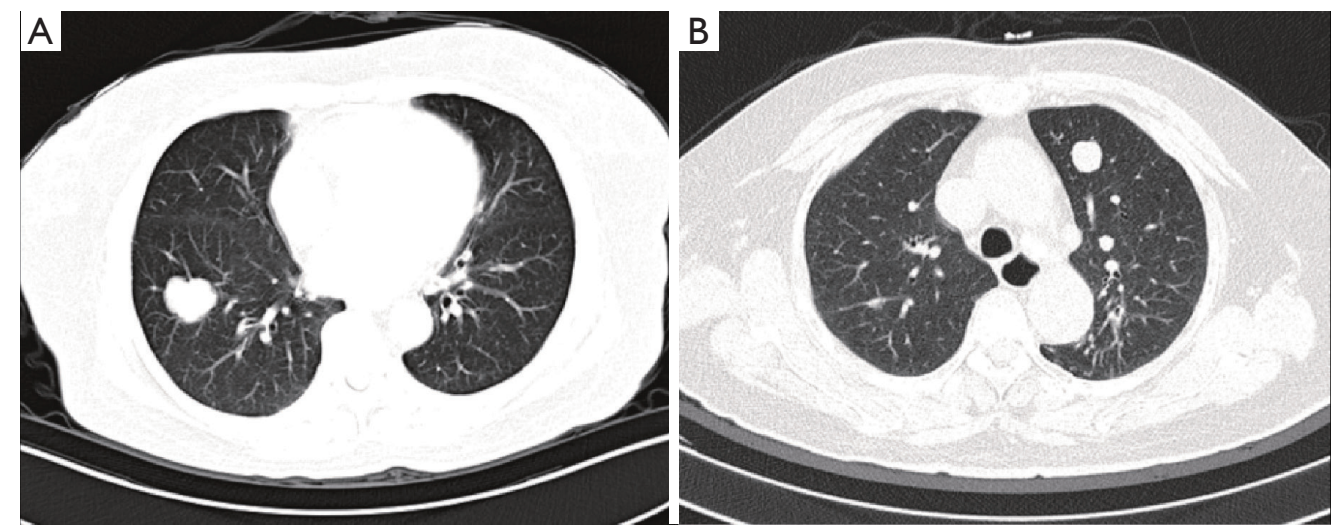

Figure 3 PPMs on CT. (A,B) PPMs showing as isolated, round, solid, and well-circumscribed nodules, with or without lobulation. PPMs, primary pulmonary meningiomas; CT, computed tomography.

manifested as an isolated, solid and well-circumscribed nodule with or without lobulation (25-29). However, one recent study reported PPMs showed as multiple cystic lesions (13). And another study reported a PPM presenting as $4.5 \mathrm{~mm}$ GGO (30). PPMs could have metabolic activity on ${ }^{18} \mathrm{~F}$-FDG PET $(25,31)$. It was usually regarded as a benign disease (28). For PPM, surgical intervention can also achieve satisfying long-term outcome reaching about 

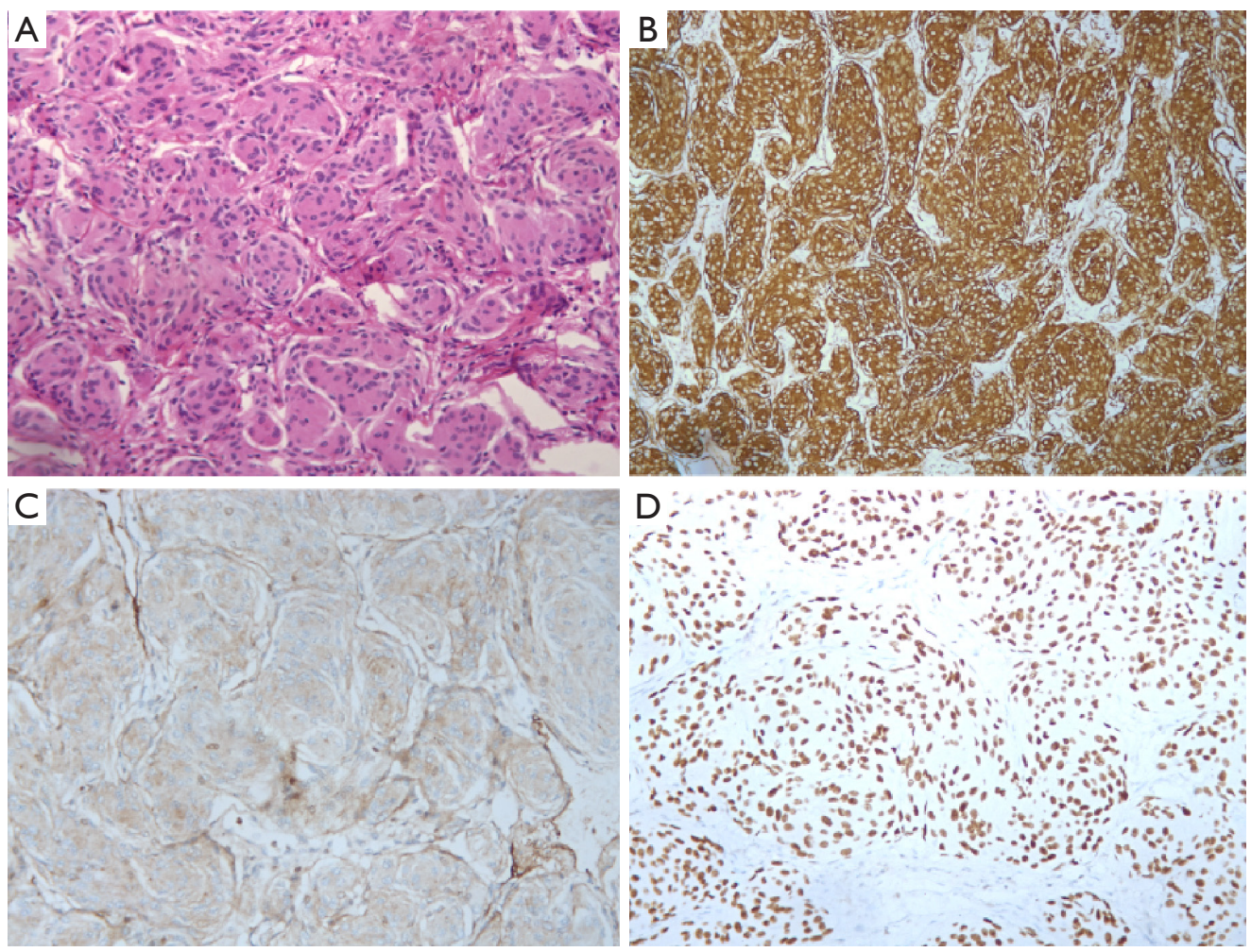

Figure 4 Histological features of PPMs. (A) Macroscopically, PPMs showed as spindle cells organized in bundles and whorls on hematoxylin-eosin staining $(\times 200)$; (B,C,D) immunohistochemically, primary pulmonary meningioma showed positive for Vimentin $(\times 100)$, EMA (×200), PR (×200). PPMs, primary pulmonary meningiomas.

Table 2 IHC outcomes of resected PPM and MPMN

\begin{tabular}{|c|c|c|c|c|}
\hline \multirow[b]{2}{*}{$\begin{array}{l}\text { IHC } \\
\text { staining }\end{array}$} & \multicolumn{2}{|c|}{ PPM (n=4) } & \multicolumn{2}{|c|}{ MPMN $\left(n=38^{\star}\right)$} \\
\hline & $\begin{array}{l}\text { Positive } \\
\text { cases/total } \\
\text { stained }\end{array}$ & Percentage & $\begin{array}{l}\text { Positive } \\
\text { cases/total } \\
\text { stained }\end{array}$ & Percentage \\
\hline EMA & $4 / 4$ & 100 & $37 / 37$ & 100 \\
\hline PR & $3 / 4$ & 75 & $30 / 35$ & 85.7 \\
\hline Vimentin & $4 / 4$ & 100 & $12 / 13$ & 92.3 \\
\hline $\mathrm{Ki}-67$ & $3 / 3$ & 100 & $15 / 15$ & 100 \\
\hline ER & $0 / 1$ & 0 & $0 / 1$ & 0 \\
\hline CD56 & $0 / 1$ & 0 & $4 / 5$ & 80 \\
\hline CD68 & $1 / 1$ & 100 & $5 / 5$ & 100 \\
\hline TTF-1 & $0 / 2$ & 0 & $5 / 14$ & 35.7 \\
\hline Syn & $0 / 1$ & 0 & $1 / 7$ & 14.3 \\
\hline $\mathrm{CHG}$ & $0 / 0$ & 0 & $2 / 4$ & 50 \\
\hline CD34 & $2 / 3$ & 66.7 & $5 / 6$ & 83.3 \\
\hline
\end{tabular}

${ }^{*}$, Thirty-eight lesions of 35 patients underwent IHC examination. IHC, immunohistochemical; PPM, primary pulmonary meningioma; MPMN, minute pulmonary meningothelial-like nodule.
24 years (32). Ohashi-Nakatani et al. had reported preoperative cytological assessment (28). In our study, one of the four cases underwent a percutaneous puncture and histological examination in local hospital. And it was confirmed as pulmonary meningioma. Thus, the lobectomy could have been avoided, and active observation could be an alternative.

One previous study suggested that MPMNs often presented in female patients and coexisted with adenocarcinoma (17). In our study, most $(68 / 73,93.2 \%)$ of cases with MPMNs were in tiny size and were resected due to the synchronous cancer-related lesions. We presumed that the real incidence of MPMNs could be underestimated. The other five cases received surgical resection due to the initial clinical diagnosis as cancer-related lesions. It is difficult to radiologically differentiate MPMNs from early-staged lung cancer, as most of the lesions were in tiny size less than $5 \mathrm{~mm}$ showing as GGO with or without mixed components (17-19). In our study, the largest one even reached $14 \mathrm{~mm}$ in diameter. In our opinion, an active observation could be essential to avoid misdiagnosis and unnecessary resection. 

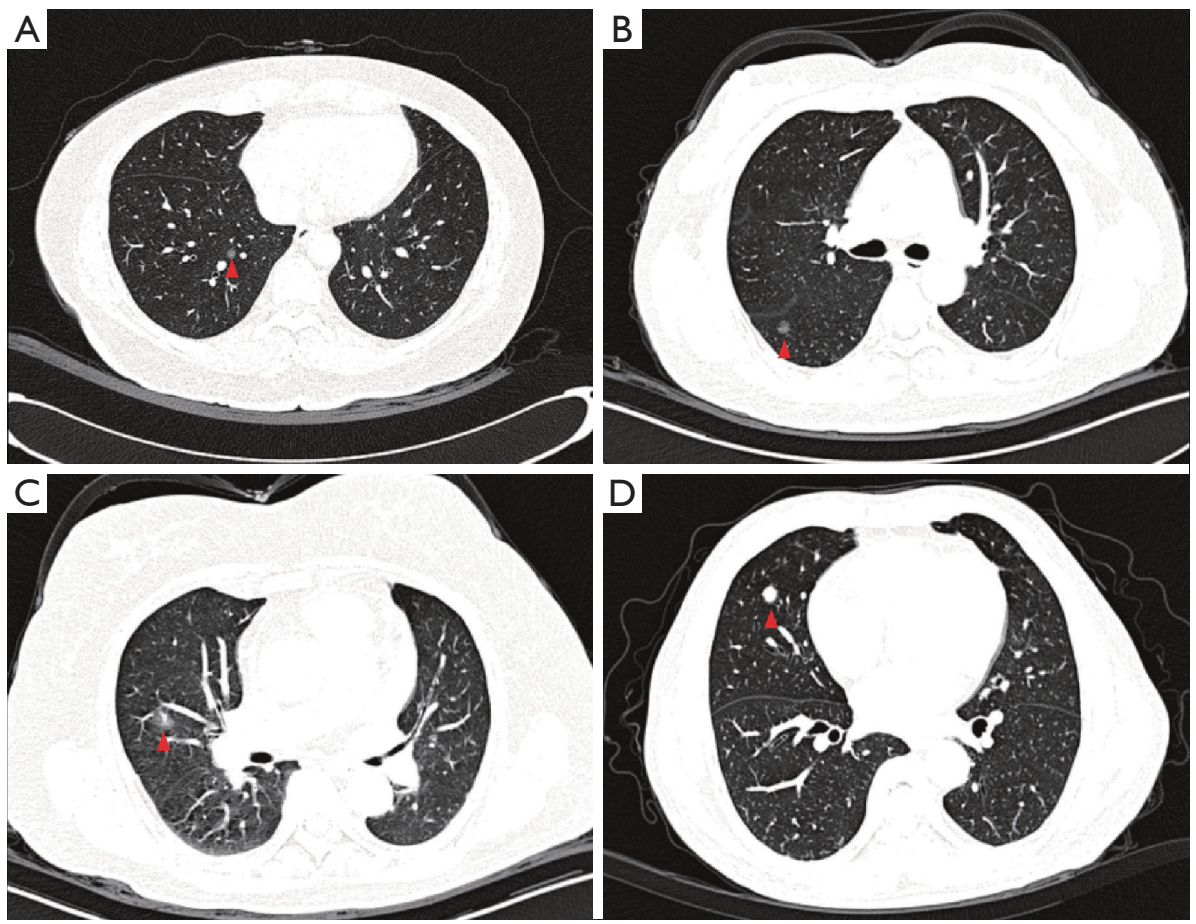

Figure 5 MPMNs on CT. (A,B,C) MPMNs showing as GGOs with or without mixed components, (D) or as solid nodules. Arrows: the small nodules in pulmonary lobes. MPMNs, minute pulmonary meningothelial-like nodules; CT, computed tomography; GGOs, groundglass opacities.
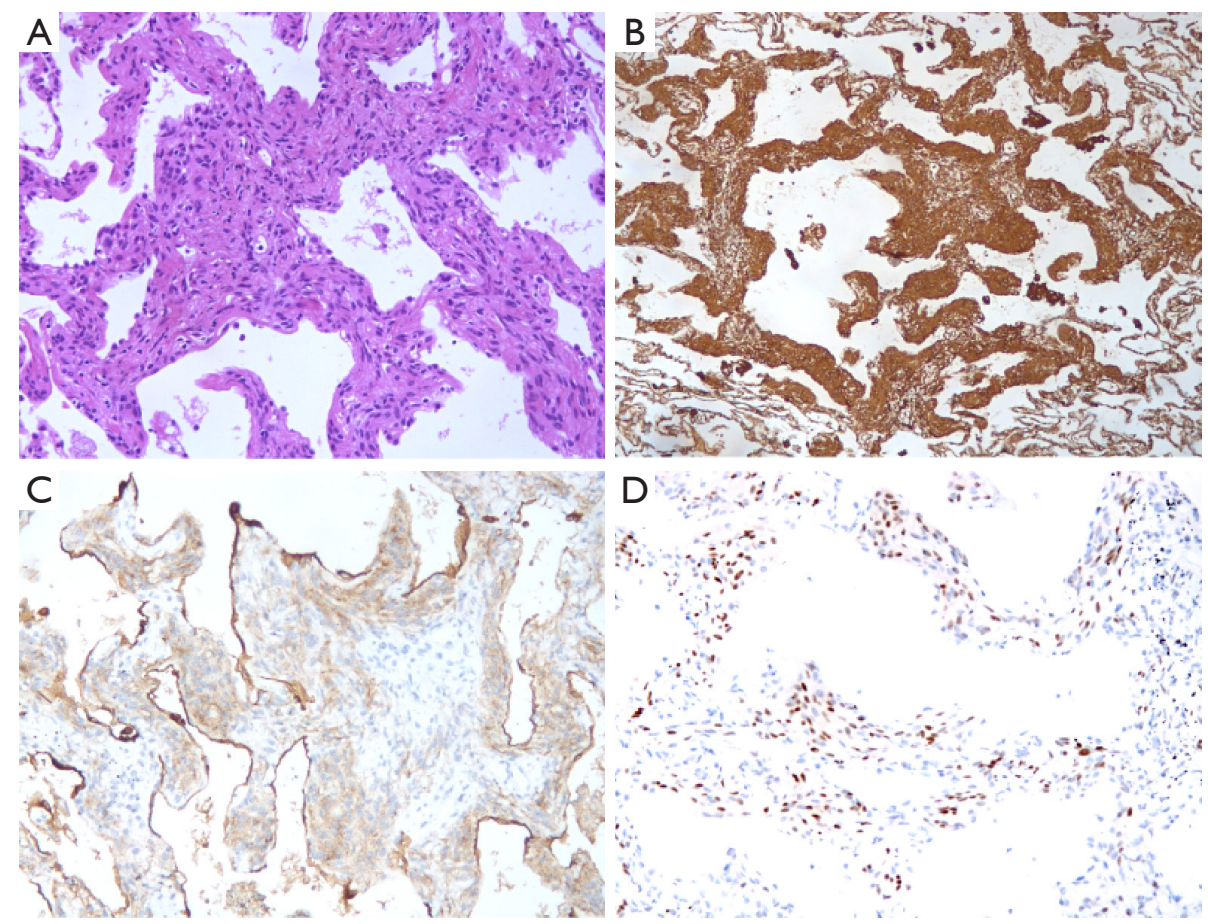

Figure 6 Histological features of MPMNs. (A) Macroscopically, MPMNs showed as some spindle-shaped cells on hematoxylin-eosin staining (×200); (B,C,D) immunohistochemically, MPMNs showed positive for Vimentin (×100), EMA (×200), PR (×200). MPMNs, minute pulmonary meningothelial-like nodules. 
Surveillance period before and after surgery

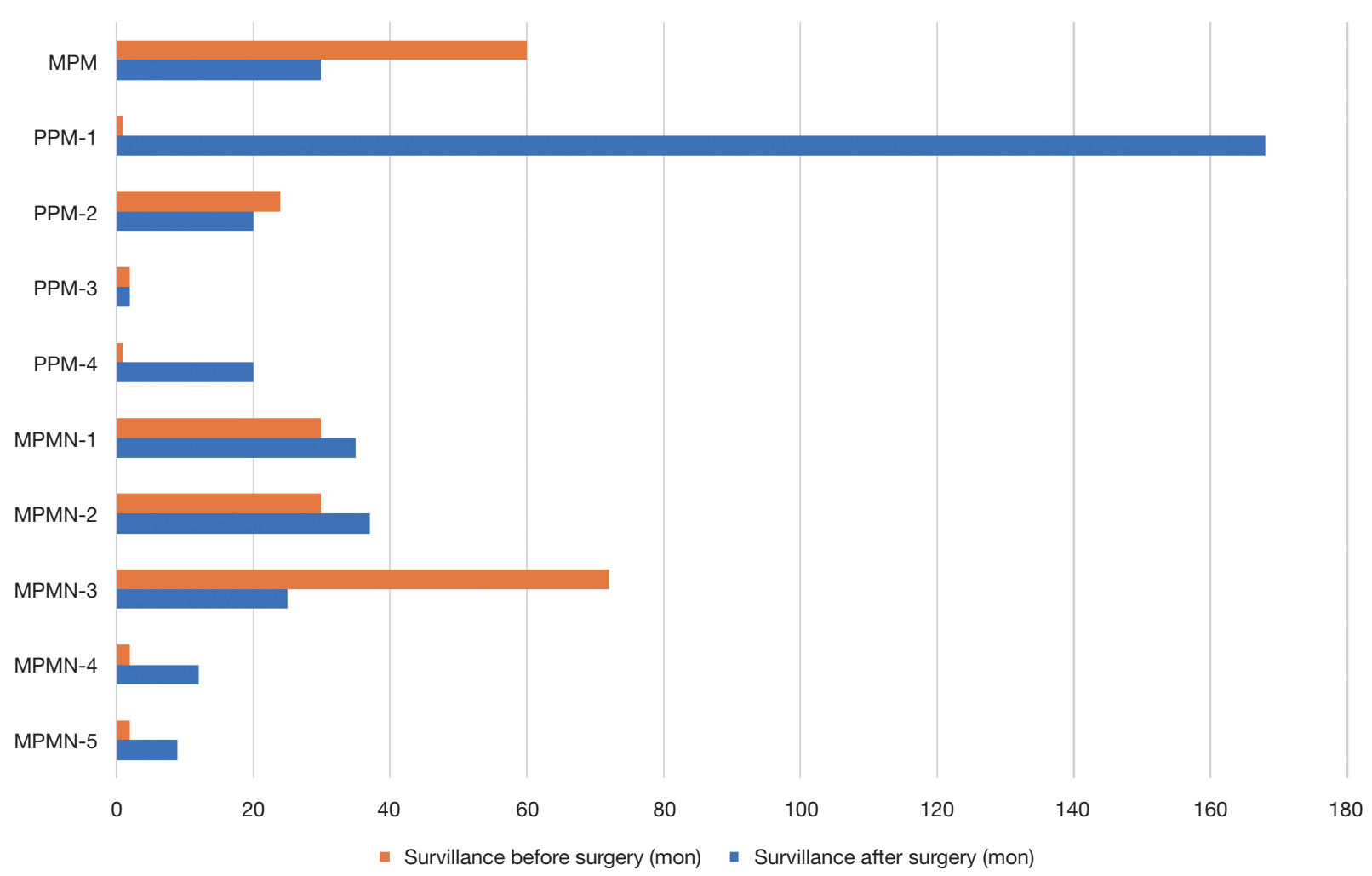

Figure 7 Surveillance periods before and after surgery of the one case with MPM, the four cases with PPMs, and the five cases with MPMNs (with no lung cancer-related lesions). MPM, metastatic pulmonary meningioma; PPMs, primary pulmonary meningiomas; MPMNs, minute pulmonary meningothelial-like nodules.

In general, radiological diagnosis of PMP is of difficulty. However, because of the characteristic histological features, percutaneous puncture is able to offer an accurate answer. As shown in Figure 7, the one case with MPM, the four case with PPM, and the five case with MPMN but no cancerrelated lesions showed a considerable surveillance before surgery and a satisfying surveillance after surgery. Thus, PMP tends be a benign disease with indolent nature. In our opinion, although surgical resection can achieve good prognosis, active observation is also an alternative due to the surgical invasiveness and the indolent nature of the disease.

There were some limitations in our study. During the studied period, more than tens of thousands of cases underwent surgical resection for pulmonary lesions in our center. However, there was no direct comparison between PMP and other pulmonary lesions in our study. But as a case series study, it contained a relatively large sample size with medical imaging and clinical management. We also proposed some undetermined questions with potential strategies. All of these might be of some importance for further improvement in radiological diagnosis and clinical management.

\section{Conclusions}

PPM has variable radiological manifestations. But it can be histologically identified by the characteristic features. Although surgery is able to achieve satisfying prognosis, active observation is an alternative in selected cases due to the surgical invasiveness and the indolent nature of the disease.

\section{Acknowledgments}

We thank Dr. Dongxian Jiang (Department of Pathology, Zhongshan Hospital Fudan University) for the kind help in the interpretation of the histological features. 
Funding: None.

\section{Footnote}

Data Sharing Statement: Available at http://dx.doi. org/10.21037/tlcr-19-699

Conflicts of Interest: All authors have completed the ICMJE uniform disclosure form (available at http://dx.doi. org/10.21037/tlcr-19-699). The authors have no conflicts of interest to declare.

Ethical Statement: The authors are accountable for all aspects of the work in ensuring that questions related to the accuracy or integrity of any part of the work are appropriately investigated and resolved. Each patient had signed the informed consent form and approved the usage of the information at the initial administration. The institutional review board of Zhongshan Hospital of Fudan University approved this study (HGBB-20191201). The study was conducted in accordance with the Declaration of Helsinki (as revised in 2013).

Open Access Statement: This is an Open Access article distributed in accordance with the Creative Commons Attribution-NonCommercial-NoDerivs 4.0 International License (CC BY-NC-ND 4.0), which permits the noncommercial replication and distribution of the article with the strict proviso that no changes or edits are made and the original work is properly cited (including links to both the formal publication through the relevant DOI and the license). See: https://creativecommons.org/licenses/by-nc-nd/4.0/.

\section{References}

1. Bray F, Ferlay J, Soerjomataram I, et al. Global cancer statistics 2018: GLOBOCAN estimates of incidence and mortality worldwide for 36 cancers in 185 countries. CA Cancer J Clin 2018;68:394-424.

2. Heuvelmans MA, Oudkerk M. Appropriate screening intervals in low-dose CT lung cancer screening. Transl Lung Cancer Res 2018;7:281-7.

3. Reyes ME, Schabath MB. Optimal lung cancer screening intervals following a negative low-dose computed tomography result. J Thorac Dis 2019;11:S1916-8.

4. McWilliams A, Tammemagi MC, Mayo JR, et al. Probability of cancer in pulmonary nodules detected on first screening CT. N Engl J Med 2013;369:910-9.
5. Ionescu DN, Sasatomi E, Aldeeb D, et al. Pulmonary meningothelial-like nodules: a genotypic comparison with meningiomas. Am J Surg Pathol 2004;28:207-14.

6. Mukhopadhyay S, El-Zammar OA, Katzenstein AL. Pulmonary meningothelial-like nodules: new insights into a common but poorly understood entity. Am J Surg Pathol 2009;33:487-95.

7. Weissferdt A, Tang X, Suster S, et al. Pleuropulmonary meningothelial proliferations: evidence for a common histogenesis. Am J Surg Pathol 2015;39:1673-8.

8. Higuchi $M$, Watanabe $M$, Inoue $T$, et al. Brief report on similar mutational changes in neurofibromatosis type 2 gene in minute pulmonary meningothelial-like nodule and meningioma of the central nervous system. Oncotarget 2018;9:36012-6.

9. Karasick JL, Mullan SF. A survey of metastatic meningiomas. J Neurosurg 1974;40:206-12.

10. Peck AG, Dedrick CG, Taft PD. Pulmonary metastases from intracerebral meningioma. Diagnosis by percutaneous needle aspiration biopsy. AJR Am J Roentgenol 1976;126:419-22.

11. Dalle Ore CL, Magill ST, Yen AJ, et al. Meningioma metastases: incidence and proposed screening paradigm. J Neurosurg 2019. [Epub ahead of print].

12. Cesario A, Galetta D, Margaritora S, et al. Unsuspected primary pulmonary meningioma. Eur J Cardiothorac Surg 2002;21:553-5.

13. Wang X, Li P, Zhou P, et al. Intrapulmonary metastasis from primary pulmonary meningioma presenting as multiple cystic lesions: a case report. BMC Pulm Med 2019;19:8.

14. Weber C, Pautex S, Zulian GB, et al. Primary pulmonary malignant meningioma with lymph node and liver metastasis in a centenary woman, an autopsy case. Virchows Arch 2013;462:481-5.

15. Korn D, Bensch K, Liebow AA, et al. Multiple minute pulmonary tumors resembling chemodectomas. Am J Pathol 1960;37:641-72.

16. Gaffey MJ, Mills SE, Askin FB. Minute pulmonary meningothelial-like nodules. A clinicopathologic study of so-called minute pulmonary chemodectoma. Am J Surg Pathol 1988;12:167-75.

17. Mizutani E, Tsuta K, Maeshima AM, et al. Minute pulmonary meningothelial-like nodules: clinicopathologic analysis of 121 patients. Hum Pathol 2009;40:678-82.

18. Peng XX, Yan LX, Liu C, et al. Benign disease prone to be misdiagnosed as malignant pulmonary nodules: Minute meningothelioid nodules. Thorac Cancer 2019;10:1182-7. 
19. Asakawa A, Horio H, Hishima T, et al. Clinicopathologic features of minute pulmonary meningothelial-like nodules. Asian Cardiovasc Thorac Ann 2017;25:509-12.

20. Rajadurai S, Thotathil Z, Biju R, et al. Extensive pulmonary metastases 13 years after initial resection of intracranial meningioma. Asian J Neurosurg 2019;14:314-7.

21. D'Aiuto M, Veronesi G, Pelosi G, et al. Two-year survival after multiple bilateral lung metastasectomies for cranial meningioma. Ann Thorac Surg 2005;80:1129-30.

22. Chiarelli M, De Simone M, Gerosa M, et al. An incidental pulmonary meningioma revealing an intracranial meningioma: primary or secondary lesion? Ann Thorac Surg 2015;99:e83-4.

23. Goldbrunner R, Minniti G, Preusser M, et al. EANO guidelines for the diagnosis and treatment of meningiomas. Lancet Oncol 2016;17:e383-91.

24. Apra C, Peyre M, Kalamarides M. Current treatment options for meningioma. Expert Rev Neurother 2018;18:241-9.

25. Incarbone M, Ceresoli GL, Di Tommaso L, et al. Primary pulmonary meningioma: report of a case and review of the literature. Lung Cancer 2008;62:401-7.

26. Satoh Y, Ishikawa Y. Primary pulmonary meningioma: ten-year follow-up findings for a multiple case, implying

Cite this article as: Lin D, Yu Y, Wang H, Fang Y, Yin J, Shen Y, Tan L. Radiological manifestations, histological features and surgical outcomes of pulmonary meningothelial proliferation: a case series and rethinking. Transl Lung Cancer Res 2020;9(4):1159-1168. doi: 10.21037/tlcr-19-699 a benign biological nature. J Thorac Cardiovasc Surg 2010;139:e39-40.

27. Kim YY, Hong YK, Kie JH, et al. Primary pulmonary meningioma: an unusual cause of a nodule with strong and homogeneous enhancement. Clin Imaging 2016;40:170-3.

28. Ohashi-Nakatani K, Shibuki Y, Fujima M, et al. Primary pulmonary meningioma: a rare case report of aspiration cytological features and immunohistochemical assessment. Diagn Cytopathol 2019;47:330-3.

29. Luo JZ, Zhan C, Ni X, et al. Primary pulmonary meningioma mimicking lung metastatic tumor: a case report. J Cardiothorac Surg 2018;13:99.

30. Juan CM, Chen ML, Ho SY, et al. Primary pulmonary meningioma simulating a pulmonary metastasis. Case Rep Pulmonol 2016;2016:8248749.

31. Meirelles GS, Ravizzini G, Moreira AL, et al. Primary pulmonary meningioma manifesting as a solitary pulmonary nodule with a false-positive PET scan. J Thorac Imaging 2006;21:225-7.

32. Moran CA, Hochholzer L, Rush W, et al. Primary intrapulmonary meningiomas. A clinicopathologic and immunohistochemical study of ten cases. Cancer 1996;78:2328-33. 